\title{
Galactosylated poly(ethylene glycol)-b-poly (I-lactide-co- $\beta$-malic acid) block copolymer micelles for targeted drug delivery: preparation and in vitro characterization
}

\author{
This article was published in the following Dove Press journal: \\ International Journal of Nanomedicine \\ 22 November 2010 \\ Number of times this article has been viewed
}

\begin{abstract}
Aili Suo'
Junmin Qian²

Yu Yao'

Wanggang Zhang ${ }^{3}$

'Department of Medical Oncology, First Affiliated Hospital of Medical School, Xi'an Jiaotong University, ${ }^{2}$ State Key Laboratory for Mechanical Behaviors of Materials, School of Materials Science and Engineering, Xi'an Jiaotong University, ${ }^{3}$ Second Affiliated Hospital of Medical School Xi'an Jiaotong University, Xi'an, China
\end{abstract}

Correspondence: Aili Suo

Department of Medical Onconology, First Affiliated Hospital of Medical School, Xi'an Jiatong University, 277 Yanta West Road, Xi'an 7I006I, People's Republic of China

Tel +862985324600

$\mathrm{Fax}+862982663453$

Email address ailisuo@gmail.com
Abstract: Biodegradable galactosylated methoxy poly(ethylene glycol)/poly(l-lactide-co$\beta$-malic acid) (Gal-PEG-b-PLMA) block copolymer micelles were successfully prepared by a solvent diffusion method, and could efficiently encapsulate doxorubicin. The Gal-PEG-b-PLMA micelles before and after doxorubicin loading were characterized by size, morphology, in vitro drug release, and in vitro cytotoxicity in HepG2 cells. Transmission electron microscopy and dynamic light scattering results showed that the empty and doxorubicin-loaded micelles were approximately spherical in shape and had mean sizes of about $72 \mathrm{~nm}$ and $85 \mathrm{~nm}$, respectively. In vitro release behavior of doxorubicin from the micelles was $\mathrm{pH}$-dependent, with obviously faster release rates at mildly acidic $\mathrm{pH} 4.5$ and 5.5 compared with physiologic pH 7.4. Methylthiazoletetrazolium assay and flow cytometric analysis indicated that the doxorubicin-loaded galactosylated micelles exhibited a greater growth-inhibitory effect on HepG2 cells than the nongalactosylated doxorubicin-loaded micelles, and induced $\mathrm{S}$ phase cell cycle arrest. Confocal laser scanning microscope observations revealed that the galactosylated micelles could be efficiently internalized by HepG2 cells through receptor-mediated endocytosis. The results suggest that Gal-PEG-b-PLMA copolymer micelles are a promising carrier system for targeted drug delivery in cancer therapy.

Keywords: polymer micelles, targeted drug delivery, doxorubicin, chemotherapy, hepatocellular carcinoma

\section{Introduction}

Hepatocellular carcinoma (HCC) is the third leading cause of cancer-related death throughout the world, ${ }^{1}$ and is strongly associated with highly prevalent hepatitis B virus infections and exposure to food contaminated with aflatoxin B1. In China alone, the estimated annual incidence of new HCC cases is more than 350,000 (approximately $55 \%$ of global HCC cases), ${ }^{2}$ and HCC is the second most common cause of cancerrelated death. Surgical resection is considered the optimal treatment approach, but is very limited in HCC patients because of high rates of recurrence and metastasis after surgery. Moreover, conventional chemotherapy is associated with toxicity to normal tissues. In addition, HCC is well known to be highly resistant to chemotherapy drugs. ${ }^{3}$ Therefore, it is of great importance to search for more effective systemic chemotherapeutic options to improve the survival rate of $\mathrm{HCC}$ patients. ${ }^{4}$ Amongst these, the rapid development of targeted drug delivery systems holds great promise for the treatment of HCC. 
Doxorubicin, a well known anticancer drug, has shown strong antitumor activity against various solid tumors, including those of the breast, bladder, lung, ovary, and liver. ${ }^{5}$ However, its clinical therapeutic potential is restricted by both low bioavailability and serious systemic toxic side effects to normal tissues. To address these problems, a great deal of effort has been focused on developing novel drug delivery systems, including liposomes, micelles, dendrimers, hyperbranched polymers, microspheres, and hydrogels. ${ }^{6-8}$ During this time, nanosized polymeric delivery systems have attracted considerable attention, because they not only control drug-release kinetics but also preferentially accumulate in solid tumors via passive targeting based on an enhanced permeability and retention effect. Amongst the biodegradable and nontoxic polymers used to prepare nanocarriers, amphiphilic block copolymers have been extensively explored and are rapidly advancing. They can self-assemble into spherical micelles exhibiting a core-shell architecture composed of hydrophobic segments as their inner core and hydrophilic segments as their outer shell in aqueous media. ${ }^{9}$ The core serves as a nanodepot for hydrophobic anticancer drugs, ${ }^{10}$ and is protected by the hydrophilic shell.

However, more and more observations show that no substantial therapeutic improvement can be sufficiently guaranteed by passive targeting. ${ }^{11}$ As we know, the sites of action of many chemotherapeutic agents are located in cells, and the efficient intracellular uptake of drugs by cancer cells is regarded as the determining step for antitumor activity. Therefore, many researchers have aimed to develop more effective site-specific delivery strategies by incorporating targeting ligands or antibodies into the surfaces of drug carriers, permitting them to be taken up by active receptormediated endocytosis. ${ }^{12-14}$ This can significantly improve the therapeutic effect of anticancer drugs and minimize toxic side effects to normal tissues. ${ }^{15}$ In active targeted therapy for HCC, galactose is considered to be a promising ligand because it can specifically recognize and bind asialoglycoprotein receptor, which is overexpressed exclusively on the surfaces of hepatic parenchymal cells. ${ }^{16,17}$ Galactose has been conjugated to drug carriers to achieve targeted chemotherapeutic or diagnostic agent delivery to HepG2 cells overexpressing the asialoglycoprotein receptor, although the precise mechanism of asialoglycoprotein receptor-mediated uptake of galactose-modified carriers into HepG2 cells remains unresolved.

The most prominent synthetic polyesters, such as polylactide, poly(lactic-co-glycolic acid), poly( $\varepsilon$-caprolactone), and their derivatives, have been extensively used in biomedical fields of controlled drug delivery, suture lines, and tissue engineering ${ }^{18}$ because they are nontoxic, biocompatible, and bioabsorbable in vivo. However, when used as drug carriers, the high crystallinity of polylactide would lead to a slow degradation rate, resulting in retarded drug release. Therefore, polylactide is usually copolymerized with functional monomers or grafted onto natural polymers to obtain ideal copolymers. Recently, some biodegradable polymers with pendant functional groups, such as hydroxyl, carboxyl, amine, and carboxylate, are attracting more interest because they may be used to conjugate drug molecules via functional covalent bonds and electrostatic actions, endowing the carriers with stimuli-responsive properties. In the past few years, poly(lactic acid-co-malic acid) (PLMA), a biocompatible and biodegradable copolyester with pendent carboxyl groups, has attracted some attention in biomedical applications. ${ }^{19-21}$ In this study, PLMA was evaluated as a potential antitumor drug carrier. We prepared targeting polymeric micelles from amphiphilic PEG-b-PLMA copolymer and investigated the feasibility of galactosylated PEG-b-PLMA (Gal-PEG-bPLMA) micelles as an active targeting carrier for the targeted delivery of doxorubicin to HepG2 cells. The doxorubicinloaded polymer micelles were characterized in terms of size and morphology, drug loading, in vitro drug-release behavior, and in vitro cell cytotoxicity.

\section{Materials and methods Materials}

The HepG2 cell line used in this study was obtained from the Cell Institute of Academia Sinica (Shanghai, China). Doxorubicin was supplied by Beijing Huafeng United Technology Co. Ltd. (Beijing, China). Fetal bovine serum and bovine calf serum were purchased from Hangzhou Sijiqing Biological Engineering Materials Co., Ltd. (Hangzhou, China). 3-(4,5dimethylthiazol-2-yl)-2,5-diphenyl tetrazolium bromide (MTT), 1-ethyl-3,3-dimethylaminopropyl carbodiimide hydrochloride (EDC), triethylamine, poly(ethylene glycol) bis(amine) $\left(\mathrm{NH}_{2}-\mathrm{PEG}-\mathrm{NH}_{2}\right.$, molecular weight 2000), and methoxy poly(ethylene glycol) (molecular weight 2000) were obtained from Sigma Co. (Shanghai, China). N-hydroxysuccinimide (NHS) was obtained from Sinopharm Chemical Reagent Co. (Shanghai, China). Dulbecco's Modified Eagle's Medium, RPMI-1640 medium, trypsin-EDTA (0.5\% trypsin, $5.3 \mathrm{mM} \mathrm{EDTA}$ ), and the antibiotic agents penicillin-streptomycin $(100 \mathrm{U} / \mathrm{mL})$ were purchased from Gibco BRL (Gaithersburg, MD). The dialysis membrane (molecular weight cutoff $3500 \mathrm{Da}$ ) was purchased from Beijing Solarbio Science and Technology Co., Ltd. (Beijing, China). 
The block copolymers were synthesized in our laboratory. All other chemicals were commercial products of analytic reagent grade, and were used as received unless otherwise stated.

\section{Synthesis of PEG-b-PLMA and Gal-PEG- b-PLMA copolymers}

Poly(1-lactide-co- $\beta$-benzyloxycarbonyl- $\beta$-propiolactone) (PLBCP), with a nominal molar ratio of about $85: 15$, and its block copolymer with methoxy-poly(ethylene glycol), ie, PEG-b-PLBCP, were synthesized as previously reported in the literature ${ }^{22,23}$ with some changes. Briefly, predetermined amounts of 1-lactide (LA) and $\beta$-benzyloxycarbonyl- $\beta$ propiolactone $(\mathrm{BCP})$ or their mixture with MPEG were placed in a polymerization tube $(8 \mathrm{~mL})$, and $0.5 \mathrm{~mL}$ of dried toluene solution containing the catalyst $\mathrm{Sn}(\mathrm{Oct})_{2}(0.1 \% \mathrm{w} / \mathrm{w})$ was added into the tube. The tube was evacuated by a vacuum pump at $30^{\circ} \mathrm{C}$ for 3 hours and then sealed. The ring-opening copolymerization was performed at $140^{\circ} \mathrm{C}$ for 24 hours. After polymerization, the reaction system was cooled to ambient temperature, and the product was dissolved in $\mathrm{CH}_{2} \mathrm{Cl}_{2}$, precipitated twice with cold diethyl ether, and dried at $35^{\circ} \mathrm{C}$ for 36 hours in a vacuum oven. Galactosylated PEG-b-PLBCP (Gal-PEG-b-PLBCP) was further synthesized by conjugating PLBCP with Gal-PEG-NH ${ }_{2}$ at room temperature for 48 hours via the carbodiimide method. Gal-PEG- $\mathrm{NH}_{2}$ was obtained from galactose and $\mathrm{NH}_{2}-\mathrm{PEG}-\mathrm{NH}_{2}$ through the condensation reaction between $\mathrm{NH}_{2}-\mathrm{PEG}-\mathrm{NH}_{2}$ and galactonic acid in the presence of NHS/EDC. Before entrapping drug or preparing micelles, PEG-b-PLBCP and Gal-PEG-b-PLBCP were transformed, respectively, into PEG-b-PLMA and GalPEG-b-PLMA by removing the protecting benzyl groups of BCP units by introducing $\mathrm{H}_{2}$ in the presence of a $\mathrm{Pd} / \mathrm{C}$ catalyst.

\section{Preparation of polymer micelles and drug loading}

Empty and doxorubicin-loaded polymer micelles were prepared by a solvent diffusion method. Briefly, $50 \mathrm{mg}$ of PEG-b-PLMA or its mixture with Gal-PEG-b-PLMA (molar ratio of 25:1) dissolved in $2 \mathrm{~mL}$ of $\mathrm{CH}_{2} \mathrm{Cl}_{2}$, with or without addition of $1-15 \mathrm{mg}$ of doxorubicin dissolved in $0.25 \mathrm{~mL}$ of dimethyl sulfoxide, was added dropwise into $10 \mathrm{~mL}$ of aqueous solution in a round-bottom flask under magnetic stirring at room temperature. The mixture was sonicated for 30 minutes to form a homogeneous micelle solution. Gentle stirring was continued under reduced pressure until the organic solvent had evaporated completely. The micelles were recovered by centrifugation at $12,000 \times \mathrm{g}$ and washed 3 times with deionized water. Dry nanomicelles were also collected by freeze drying.

To determine drug loading content and encapsulation efficiency, doxorubicin-loaded micelles were separated by centrifugation at $12,000 \times \mathrm{g}$, and the exact doxorubicin concentration in the solutions was determined by ultravioletvisible spectrometry (Ultraviolet-visible Spectrophotometer, Puxi Scientific Instruments, Beijing, China) by monitoring the characteristic absorbance of doxorubicin at $480 \mathrm{~nm}$ using a calibration curve. The calibration curve of the ultraviolet-visible absorbency was generated with a known doxorubicin concentration range of $0.01-45 \mu \mathrm{g} / \mathrm{mL}$. Drug loading content was expressed as the percentage of drug amount loaded into micelles with respect to the total amount of drug-loaded micelles. Encapsulation efficiency was defined as the percentage of the drug amount incorporated into micelles with respect to the initially added drug amount to the formulation. All samples were analyzed in triplicate.

\section{Micelle characterization}

The morphology of the prepared copolymeric micelles was characterized by transmission electron microscopy (TEM, JEM-200CX, Jeol Co., Japan). A drop of diluted micellar solution $(0.1 \mathrm{mg} / \mathrm{mL})$ was placed on a copper TEM grid, and observed at an accelerating voltage of $80 \mathrm{kV}$. The micelle size and size distribution were measured by dynamic light scattering using a Zetasizer Nano-ZS size analyzer (Malvern Instruments Ltd., Malvern, UK). Measurements were carried out in double-distilled $\mathrm{H}_{2} \mathrm{O}$ at $25^{\circ} \mathrm{C}$ and at a fixed wavelength of $633 \mathrm{~nm}$.

The critical micelle concentration of the block polymers was determined by the pyrene method. ${ }^{24}$ The concentration of polymer solutions was varied from $0.02 \mu \mathrm{g} / \mathrm{mL}$ to $0.2 \mathrm{mg} / \mathrm{mL}$, and the final concentration of fluorescence probe pyrene was fixed at $0.2 \mu \mathrm{g} / \mathrm{mL}$. The fluorescence spectra were recorded using an LS55 spectrometer (Perkin-Elmer, Waltham, MA) with an excitation wavelength of $350 \mathrm{~nm}$. Emission fluorescence at 372 and $383 \mathrm{~nm}$ was monitored. Dynamic light scattering and critical micelle concentration measurements were done in triplicate for each sample, and the results were taken as the average of three measurements.

\section{In vitro release of doxorubicin from polymer micelles}

In vitro release behavior of doxorubicin from PEG-bPLMA micelles (about 8\% drug-loaded samples) was 
observed for 72 hours in phosphate-buffered saline at different $\mathrm{pH}(4.5,5.5$, and 7.4) using a dialysis-diffusion method. Briefly, doxorubicin-loaded micelles were diluted with corresponding phosphate-buffered saline to give the desired concentration $(2 \mathrm{mg} / \mathrm{mL}, 0.5 \mathrm{~mL})$ and sealed in a dialysis bag, and the dialysis bag was immersed in $10 \mathrm{~mL}$ of the release buffer in a $15 \mathrm{~mL}$ capped centrifuge tube. The centrifuge tubes were placed in an incubator shaker with gentle shaking at $37^{\circ} \mathrm{C}$. At predetermined time intervals, $1 \mathrm{~mL}$ of release buffer was withdrawn from the tube and replaced with the same amount of fresh buffer. The amount of doxorubicin released in the collected buffer samples was determined by ultraviolet-visible spectrophotometric analysis. The release experiments were conducted in triplicate, and the results presented are average data.

\section{In vitro cell cytotoxicity}

For all the cell culture studies, HepG2 cells were used as a model tumor cell line and maintained in RPMI1640 medium with $10 \%$ fetal bovine serum, $0.1 \%$ nonessential amino acids, and $100 \mathrm{U} / \mathrm{mL}$ penicillin-streptomycin at $37^{\circ} \mathrm{C}$ in a humidified atmosphere containing $5 \% \mathrm{CO}_{2}$. The in vitro anticancer effect (cytotoxicity) of the drug-loaded micelles was evaluated using the MTT assay. Briefly, HepG2 cells at logarithmic growth phase were separately seeded in 96-well plates at a seeding density of $1 \times 10^{4}$ cells/well and incubated for 24 hours to allow the cells to attach, followed by washing with ice-cold phosphate-buffered saline three times and replacing with fresh medium. Thereafter, the cells were treated with free doxorubicin, doxorubicin-loaded PEG-b-PLMA micelles, and Gal-PEG-b-PLMA micelles. The equivalent concentration of doxorubicin in the three formulations was set at $0.05-40 \mu \mathrm{g} / \mathrm{mL}$. After the predetermined incubation time, $20 \mu \mathrm{L}$ MTT solution $(5 \mathrm{mg} / \mathrm{mL}$ in phosphate-buffered saline) was added to each well, and four hours later each well was replaced with dimethyl sulfoxide to ensure solubilization of formazan crystals. Cell viability was quantified by measuring absorbance at $570 \mathrm{~nm}$ on a microplate reader (EL310, Bio-Tek Instruments Inc., Winooski, VT), calculated and expressed as a percentage of the absorbance in control cells compared with that in the treated cells.

\section{Intracellular uptake of doxorubicin}

To observe the intracellular doxorubicin distribution in micelles, the HepG2 cells were seeded in Lab-Tek ${ }^{\circledR}$ eightwell chambers (Nalgene, Nunc Inc., Denmark) at a density of $5.0 \times 10^{4}$ cells/well and cultured overnight, followed by coincubation with the three doxorubicin formulations (equivalent doxorubicin concentration $5 \mu \mathrm{g} / \mathrm{mL}$ ) for 12 hours. The treated cells were washed three times with ice-cold phosphate-buffered saline ( $\mathrm{pH} 7.4$ ), fixed with $4 \%$ paraformaldehyde in $95 \%$ ethanol at $-20^{\circ} \mathrm{C}$ overnight, and examined by a confocal laser scanning microscope at excitation and emission wavelengths of 490 and $560 \mathrm{~nm}$, respectively. The HepG2 cells were washed once again with phosphate-buffered saline before flow cytometry analysis (FACScan flow cytometry, BD Biosciences, Franklin Lakes, $\mathrm{NJ}$ ). The experiments were performed in triplicate and the data were analyzed using the FAC station software program.

\section{Statistical analysis}

Results were expressed as mean \pm standard deviation based on three independent measurements. Statistical analyses were performed by one-way analysis of variance to determine differences among the measured properties of each group using a statistical program (SPSS 10; SPSS Inc, Chicago, IL). Statistical significance was defined as a probability level of $P<0.05$ in all cases.

\section{Results and discussion Synthesis of Gal-PEG-b-PLMA copolymer}

The schematic synthesis procedure for Gal-PEG-b-PLMA copolymers is shown in Figure 1. The ring-opening copolymerization of two cyclic aliphatic lactone monomers, LA and $\mathrm{BCP}$, could proceed smoothly in the absence or presence of MPEG-OH at $140^{\circ} \mathrm{C}$ using $\mathrm{Sn}(\mathrm{Oct})_{2}$ as a catalyst, and the removal of protecting benzyl groups in $\mathrm{BCP}$ units yielded the corresponding PLMA or PEG-b-PLMA copolymer. Their physical properties are summarized in Table 1. The molecular weight of PLMA was Mn $11.5 \mathrm{kDa}$, with a polydispersity of 1.32. Gal-PEG-b-PLMA was further synthesized from GalPEG-NH $\mathrm{N}_{2}$ and PLMA in anhydrous dimethyl sulfoxide via the condensation reaction between the amino groups of GalPEG-NH $\mathrm{N}_{2}$ and the carboxylic groups of PLMA in the presence of NHS/EDC. When the copolymerization of LA and $\mathrm{BCP}$ was performed in the presence of PEG, the feed molar ratio of PEG to the two monomers was set at 1.39:85:15. The final copolymer had a PEG/LA/BCP molar ratio of $1.4: 86: 14$, which was close to the ratio in feed, whereas, in the absence of PEG, the LA/BCP molar ratio of PLMA copolymer was $87: 13$, and also approximated the feed molar ratio of $85: 15$. These findings indicate that these two cyclic ester monomers are capable of copolymerizing in the absence 
<smiles>CCOC(=O)C1CC(=O)O1</smiles>

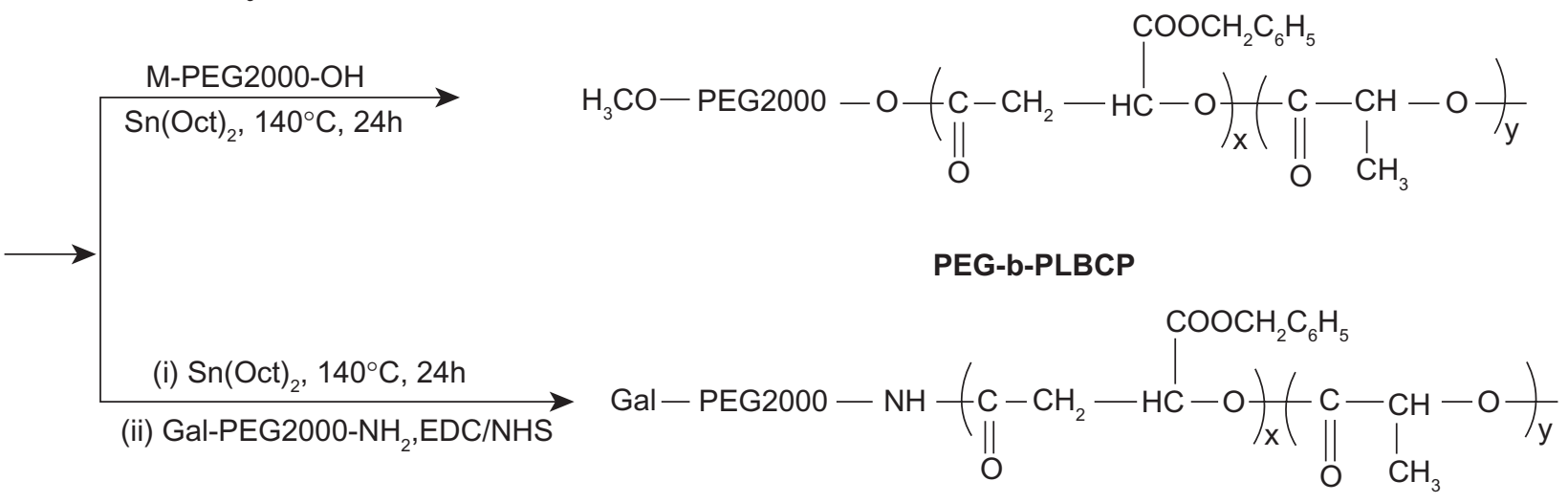

Gal-PEG-b-PLBCP

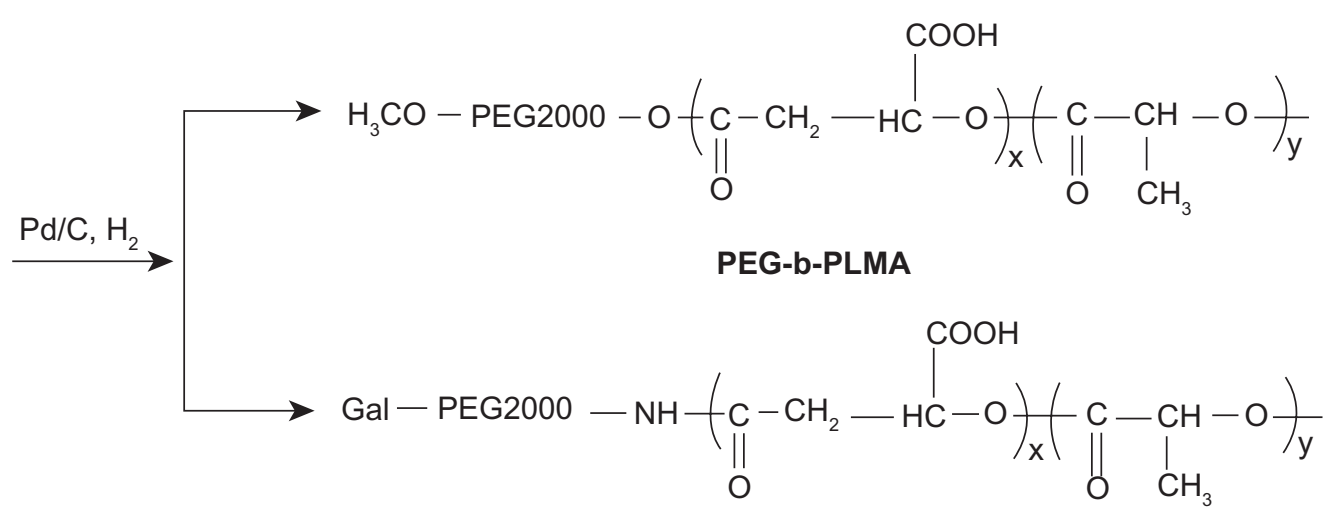

\section{Gal-PEG-b-PLMA}

Figure I Synthesis scheme of PEG-b-PLMA and Gal-PEG-b-PLMA copolymers.

Abbreviations: PEG-b-PLMA, methoxy poly(ethylene glycol)/poly(l-lactide-co- $\beta$-malic acid) block copolymer; Gal-PEG-b-PLMA, galactosylated methoxy poly(ethylene glycol)/poly(l-lactide-co- $\beta$-malic acid) block copolymer.

or presence of PEG to produce PLMA and PEG-b-PLMA copolymers.

\section{Preparation and characterization of polymer micelles}

In general, the micellization of amphiphilic block copolymers in a selective solvent of one of the blocks is a typical feature of their colloidal properties. In the present study, when amphiphilic PEG-b-PLMA copolymer was dissolved in an aqueous solution that was a thermodynamically good solvent for PEG block and a precipitant for PLMA block, the copolymer chains could associate reversibly to form micellar aggregates, in which the hydrophobic PLMA cores were surrounded by hydrophilic PEG shells. Therefore, the hydrophobic doxorubicin could be incorporated into micelles through the partitioning of doxorubicin within the

Table I Physical properties of PLMA and PEG-b-PLMA copolymers

\begin{tabular}{lllll}
\hline & $\begin{array}{l}\text { Molar ratio in feed } \\
\text { (PEG/LA/BCP) }\end{array}$ & $\begin{array}{l}\text { Molar ratio in copolymer } \\
\text { (PEG/LA/MA) }\end{array}$ & Yield (\%) & Mn ('H NMR) \\
\hline PLMA & $0: 85: 15$ & $0: 87: 13$ & 92 & 11500 \\
PEG-b-PLMA & $1.39: 85: 15$ & $1.4: 86: 14$ & 95 & 1.32 \\
\hline
\end{tabular}

Abbreviations: PLMA, poly(l-lactide-co- $\beta$-malic acid); PEG-b-PLMA, methoxy poly(ethylene glycol)/poly(l-lactide-co- $\beta$-malic acid) block copolymer. 
cores of PEG-b-PLMA micelles formed by self-assembly of the copolymer.

The size and morphology of empty and doxorubicinloaded PEG-b-PLMA micelles were investigated by dynamic light scattering and TEM. Figure 2 shows the typical
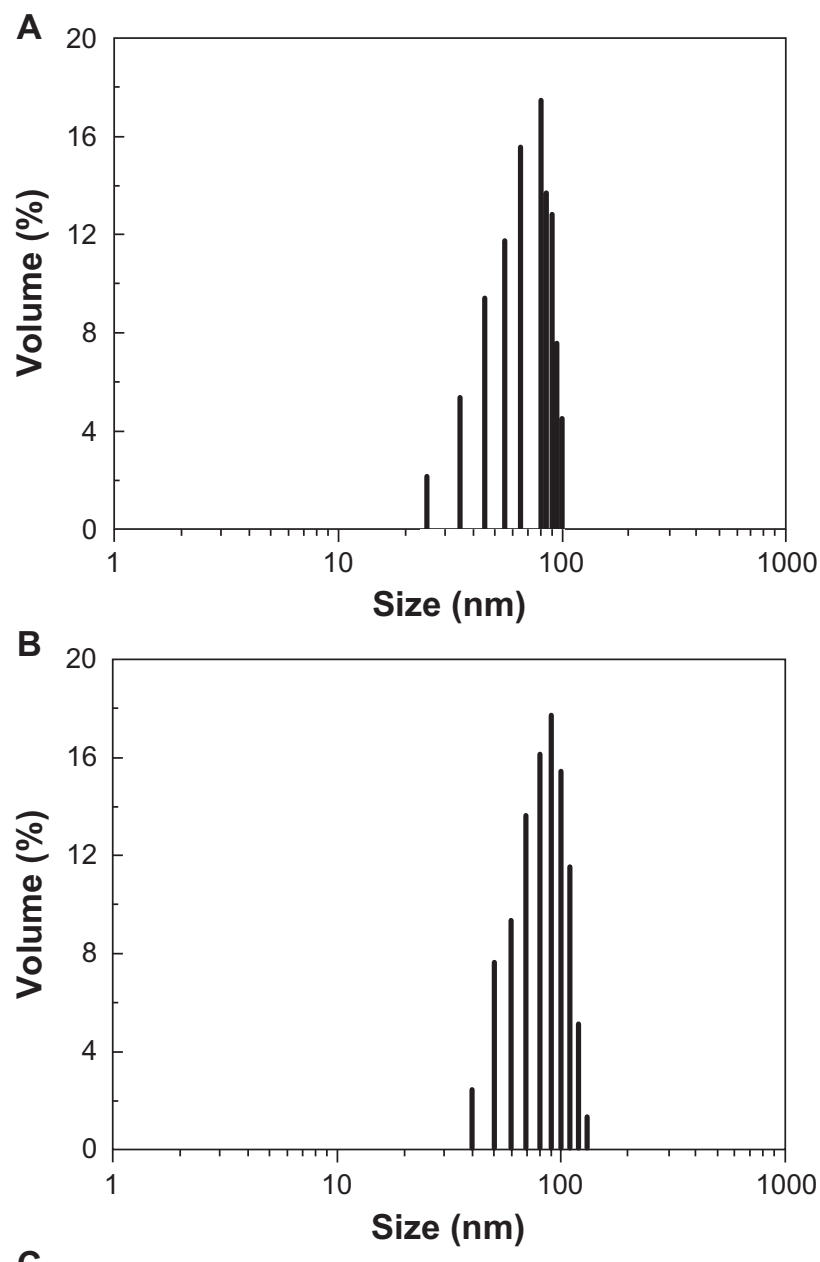

C

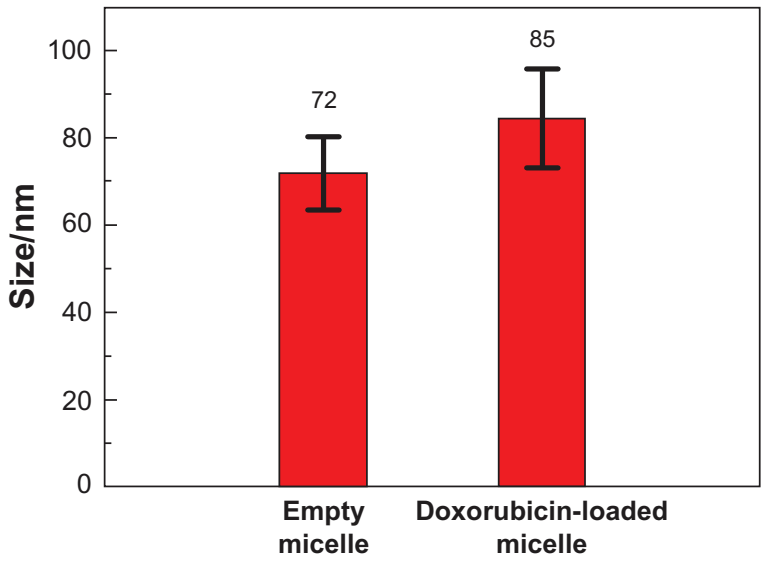

Figure 2 Size distributions of (A) empty, (B) doxorubicin-loaded Gal-PEG-b-PLMA micelles, and $(\mathbf{C})$ their mean sizes measured by dynamic light scattering method. Abbreviation: Gal-PEG-b-PLMA, galactosylated methoxy poly(ethylene glycol)/ poly(l-lactide-co- $\beta$-malic acid) block copolymer. size distribution profiles and mean sizes of empty and doxorubicin-loaded PEG-b-PLMA micelles. As shown in Figure 2, empty and doxorubicin-loaded micelles exhibit narrow unimodal size distributions, and the corresponding mean sizes are about $71.9 \pm 8.4 \mathrm{~nm}$ and $84.5 \pm 11.4 \mathrm{~nm}$, respectively. The dynamic light scattering result indicates that the entrapped doxorubicin molecules in the hydrophobic PLMA inner cores increased the mean size of the micelles. The TEM images of empty and drug-loaded PEG-b-PLMA micelles (as shown in Figure 3) indicate that the micelles had approximately spherical morphology and a smooth surface without any aggregation, and the average sizes of the micelles before and after doxorubicin loading were about $61 \mathrm{~nm}$ and $70 \mathrm{~nm}$, respectively, which were smaller than those determined by dynamic light scattering. The most probable reason for this is
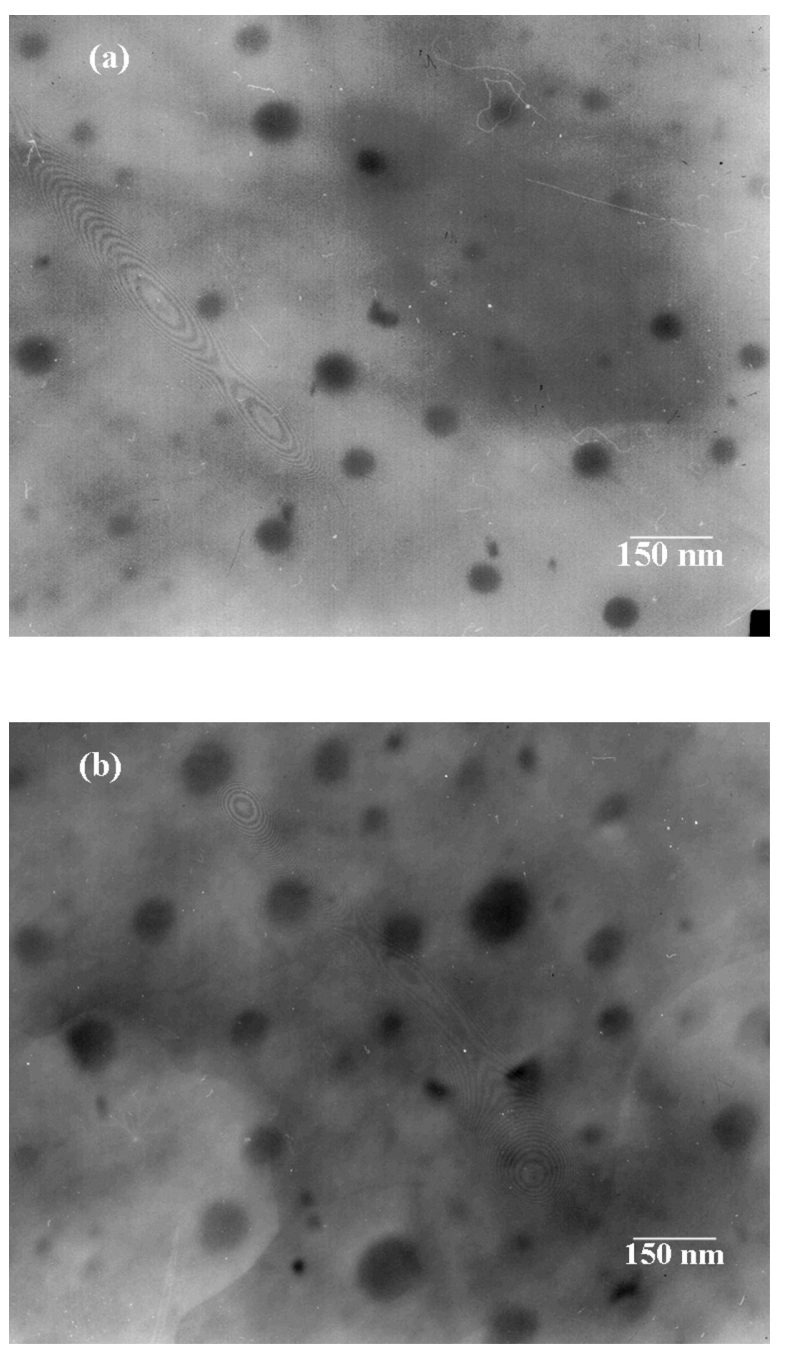

Figure 3 Transmission electron microscopic images of $(\mathbf{A})$ empty and (B) doxorubicin loaded Gal-PEG-b-PLMA micelles.

Abbreviation: Gal-PEG-b-PLMA, galactosylated methoxy poly (ethylene glycol)/ poly (I-lactide-co- $\beta$-malic acid) block copolymer. 
the shrinkage of the PEG shell caused by the evaporation of water during drying before TEM observations. As we know, the particle size and surface characteristics of micelles have a significant impact on the in vivo fate of a micelle drug delivery system. Generally, micelles of $<100 \mathrm{~nm}$ diameter with hydrophilic surfaces have a longer circulation time in blood and can selectively accumulate at tumor sites as a result of an enhanced permeability and retention effect ${ }^{25}$ or be preferentially taken up by tumor cells via receptor-mediated endocytosis, ${ }^{26}$ because they readily escape from monocytes and the reticuloendothelial system.

Doxorubicin has demonstrated significant activity in clinical applications against a wide variety of tumors. However, its serious side effects in normal tissues restrict its wide application. Therefore, the incorporation of doxorubicin into the cores of PEG-b-PLMA micelles would not only increase solubility of the drug but also reduce side effects. During the experiments, the amount of doxorubicin incorporated into the polymer micelles increased as the initial feed weight ratio of doxorubicin to copolymer increased. The drug loading content and encapsulation efficiency of doxorubicin in PEG-PLMA micelles could vary from $2.58 \% \pm 0.44 \%$ and $28.1 \%$ to $16.4 \% \pm 0.58 \%$ and $92.5 \%$, respectively, by adjusting the feed drug-to-polymer weight ratio. The reason for the high drug-loading capacity is that doxorubicin could be encapsulated into the PEG-b-PLMA micelles through the cooperative hydrophobic and electrostatic (hydrogen bond) interactions between the PLMA block with pendant carboxylic groups and doxorubicin with an amine group. However, when the feed weight ratio of doxorubicin to polymer was higher than $0.16: 1$, little aggregation was found, and drug-loading efficiency gradually decreased due to the hydrophobic nature of doxorubicin. Thus, the initial feed weight ratio of doxorubicin to polymer was fixed at $0.11: 1$ for all samples, and the actual drug loading content and encapsulation efficiency were $7.93 \% \pm 0.56 \%$ and $83.2 \% \pm 3.7 \%$, respectively. In addition, the critical micelle concentration values of PEG-b-PLMA and Gal-PEG-b-PLMA were as low as 0.9 and $1.1 \mu \mathrm{g} / \mathrm{mL}$, respectively. The results suggested that the micelles had high stability and would have the ability to maintain their integrity even with strong dilution in the body.

\section{In vitro release profile of doxorubicin from micelles}

Characterization of the in vitro release of doxorubicin from PEG-b-PLMA micelles is important to assess the stability of doxorubicin incorporated into the micelle. The in vitro release behavior of doxorubicin from PEG-b-PLMA micelles was investigated by the dialysis method at $37^{\circ} \mathrm{C}$ and at $\mathrm{pH} 4.5,5.5$, and 7.4, as shown in Figure 4. The results demonstrate that the micelles exhibited $\mathrm{pH}$-dependent release behavior, whereby much faster release of doxorubicin took place at an acidic $\mathrm{pH} 4.5$ and 5.5 than at physiologic pH 7.4. For instance, at least 30,20 , and $10 \%$ of the initial doxorubicin amount was released within 5 hours at $\mathrm{pH} 4.5$, 5.5 , and 7.4, respectively, while within 48 hours, about $78 \%, 67 \%$, and $31 \%$ of doxorubicin was released at $\mathrm{pH} 4.5$, 5.5 , and 7.4, respectively. It should be noted that over $65 \%$ of the doxorubicin was released at a lower $\mathrm{pH}$ of 4.5 after 24 hours. These results suggest that rapid drug release at endosomal $\mathrm{pH}$ renders the $\mathrm{pH}$-responsive micelles promising as smart nanovehicles for triggered release of lipophilic anticancer drugs. The in vitro release profile depicted in Figure 4 indicates two-phase release behavior, ie, a burst release during the initial several hours, followed by a slow sustained-release phase, which can be described by the zeroorder equation and Higuchi's equation, respectively. A burst release of $15 \%-45 \%$ doxorubicin occurred in the first 10 hours, and is attributed to those drug molecules existing near the micellar surface and on the interface of the micelle hydrophobic core and hydrophilic shell, which easily diffuse within the first few hours of incubation via both passive diffusion and hydration, whereas the drug incorporated into the inner core compartment inside the micelles would be responsible for sustained release after the first 10 hours. The results indicated that release of drug from the micelles was diffusion-controlled.

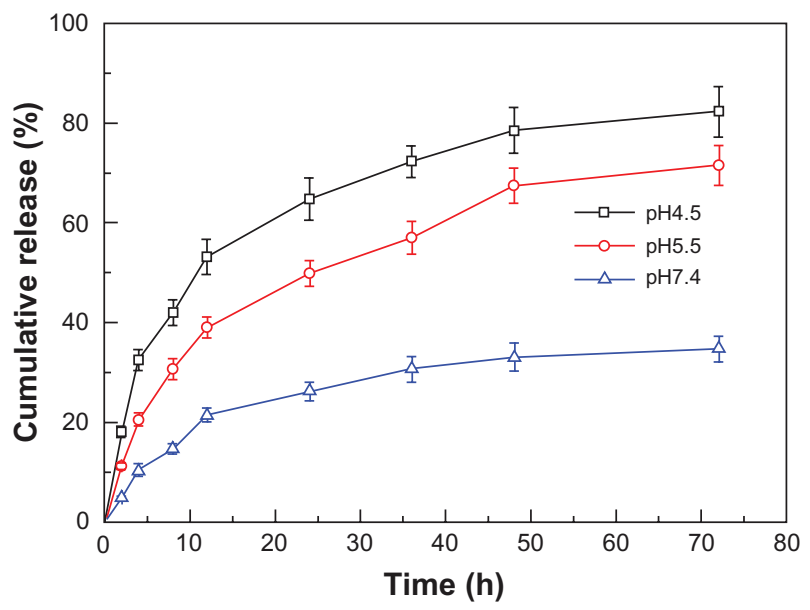

Figure 4 In vitro release behavior of doxorubicin from PEG-b-PLMA micelles at $\mathrm{pH} 4.5,5.5$, and 7.4. Each point and error bar represents mean \pm standard deviation $(n \pm 3)$.

Abbreviation: PEG-b-PLMA, methoxy poly(ethylene glycol)/poly(l-lactide-co- $\beta$ malic acid). 
The mechanism of $\mathrm{pH}$-responsive doxorubicin release might involve protonation of the amine group of doxorubicin and the ionization degree of the carboxylic groups in the PLMA block (pKa value of PLMA copolymer 3.81). Under physiologic conditions, the hydrophobic and electrostatic interactions between PLMA and doxorubicin were relatively strong, and could prevent rapid drug release. However, under mildly acidic conditions, the protonation degree of doxorubicin increases, and the ionization degree of the carboxyl groups in PLMA decreases, which leads to weaker interaction between doxorubicin and PLMA. At the same time, repulsion between protonated doxorubicin molecules might facilitate doxorubicin to diffuse out of the micelles. In addition, the carboxylic acid groups could catalyze the hydrolysis degradation of PLMA at low $\mathrm{pH}$ values, namely, PLMA erosion. The above three mechanisms could lead to rapid in vitro release.

\section{In vitro cytotoxicity in HepG2 cells}

The cytotoxic mechanism of doxorubicin involves induction of DNA damage, inhibition of cell proliferation, cell cycle status, and cell death. ${ }^{27}$ To determine the biologic effects of doxorubicin in HepG2 cells, the cytotoxicity of serial equivalent concentrations of doxorubicin in the three different formulations (free doxorubicin, doxorubicin-loaded PEG-b-PLMA micelles, and doxorubicin-loaded Gal-PEG-b-PLMA micelles) to HepG2 cells was investigated by MTT assay after 24 hours of incubation, as shown in Figure 5. Empty PEG-b-PLMA micelles were used as a negative control. HepG2 cells were reported to overexpress asialoglycoprotein receptors on their surface and to be sensitive to doxorubicin. ${ }^{28,29}$ Figure 5 shows clearly that the viability of HepG2 cells gradually decreased

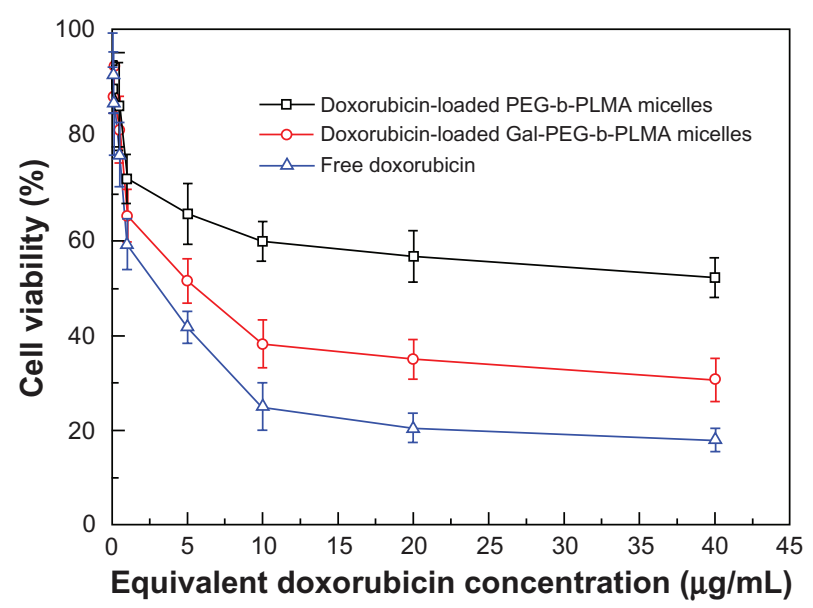

Figure 5 In vitro cytotoxicity of free doxorubicin, doxorubicin-loaded PEG-b-PLMA micelles and doxorubicin-loaded Gal-PEG-b-PLMA micelles in HepG2 cells. Data are shown as mean \pm standard deviation $(n=3)$.

Abbreviations: PEG-b-PLMA, methoxy poly(ethylene glycol)/poly(l-lactide-co- $\beta$ malic acid); Gal-PEG-b-PLMA, galactosylated methoxy poly(ethylene glycol)/poly (I-lactide-co- $\beta$-malic acid). with increasing doxorubicin concentration. Significant differences in cell cytotoxicity were observed among the three formulations at high doxorubicin doses $(>0.5 \mu \mathrm{g} / \mathrm{mL})$.

In the case of PEG-b-PLMA micelles without galactose groups, the viability of HepG2 cells was as high as $73.2 \%$ when the concentration of doxorubicin was $1 \mu \mathrm{g} / \mathrm{mL}$. In contrast, galactosylated micelles exhibited significantly superior cytotoxicity against HepG2 cells, and the cell survival rate was $65.4 \%$. The cells treated with the doxorubicin-loaded Gal-PEGb-PLMA micelles exhibited lower survival rates than those treated with doxorubicin-loaded PEG-b-PLMA micelles. This result implies that galactosylated micelles could be effectively transferred into HepG2 cells via galactose receptor-mediated endocytosis, producing more efficient killing of cancer cells.

Apoptosis of HepG2 cells induced by the three formulations was also observed by flow cytometry. The respective doxorubicin concentration was adjusted to $5 \mu \mathrm{g} / \mathrm{mL}$, and the cells were treated with the test solutions for 12 hours. Figure 6 shows the cell cycle profiles of HepG 2 cells before and after the doxorubicin treatments. As shown in Figure 6, significant $\mathrm{S}$ phase cell cycle arrest is observed in all three doxorubicin treatment groups. The percentages of cells in the S phase are $39.3 \% \pm 1.84 \%$ for the free doxorubicin group, $53.2 \% \pm 3.1 \%$ for the doxorubicin-loaded PEG-b-PLMA micelle group, and $47.6 \% \pm 2.1 \%$ for the doxorubicinloaded Gal-PEG-b-PLMA micelle group, respectively, and are much higher than the $27.2 \% \pm 0.86 \%$ recorded for the control group. This result is accompanied by lower percentages of $\mathrm{G} 0 / \mathrm{G} 1$ phase cells and higher percentages of $\mathrm{G} 2 / \mathrm{M}$

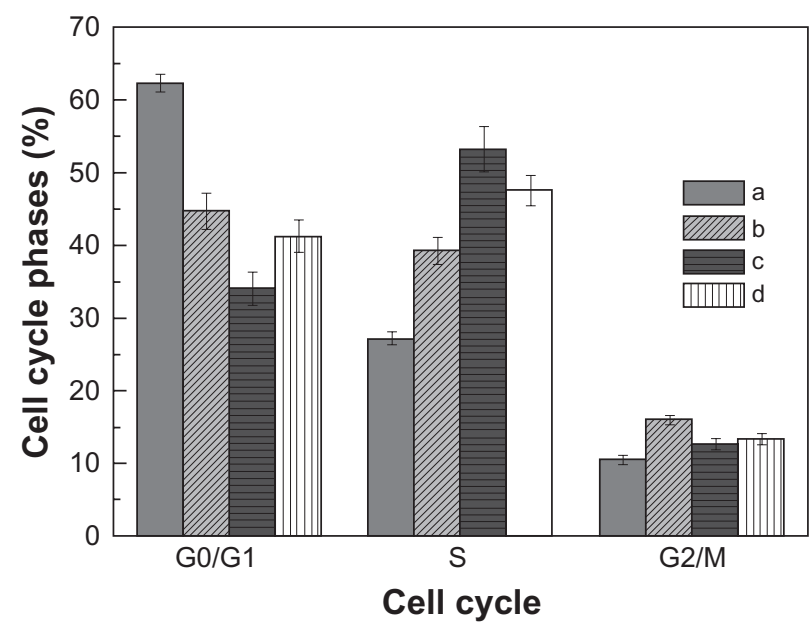

Figure 6 The cell cycle profiles of HepG2 cells before and after doxorubicin treatment for 12 hours: A) control group, B) free doxorubicin group, C) doxorubicin-loaded PEG-b-PLMA micelle group, and D) doxorubicin-loaded Gal-PEG-b-PLMA micelle group. The data represent mean \pm standard deviation where $n=3$.

Abbreviations: PEG-b-PLMA, methoxy poly(ethylene glycol)/poly(l-lactide-co- $\beta$ malic acid); Gal-PEG-b-PLMA, galactosylated methoxy poly(ethylene glycol)/poly (I-lactide-co- $\beta$-malic acid). 
phase cells in the three doxorubicin-treated groups. It is also noted that the percentages of S phase cells in the two doxorubicin-loaded micelle groups are higher than those in the free doxorubicin group. However, no obvious cell cycle changes were found among the three doxorubicin
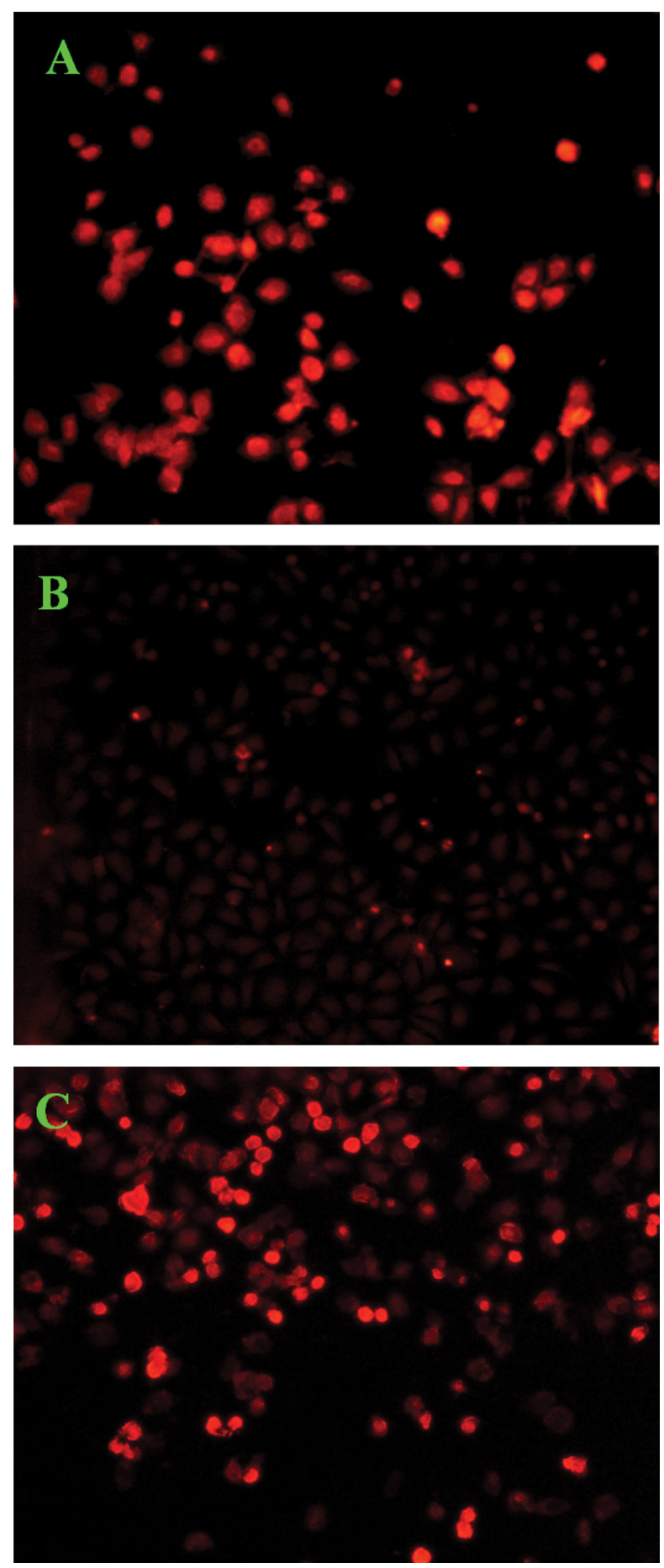

Figure 7 Confocal laser scanning microscopic images of HepG2 cells $\left(5 \times 10^{4} \mathrm{cells} / \mathrm{mL}\right)$ after 12 hours of in vitro exposure to (A) free doxorubicin, (B) doxorubicin-loaded PEG-b-PLMA micelles, and (C) doxorubicin-loaded Gal-PEG-b-PLMA micelles. Abbreviations: PEG-b-PLMA, methoxy poly(ethylene glycol)/poly (I-lactide-co- $\beta$ malic acid); Gal-PEG-b-PLMA, galactosylated methoxy poly(ethylene glycol)/poly(llactide-co- $\beta$-malic acid). formulations. Therefore, doxorubicin released from micelles can restore the cell cycle in HepG2 cells, ie, blocking cells at the $\mathrm{S}$ phase and retarding $\mathrm{G} 2 / \mathrm{M}$ phase progression.

\section{Targeting activity of Gal-PEG-b-PLMA micelles in HepG2 cells}

The intracellular delivery of doxorubicin into tumor cells is essential to its antitumor activity, because its site of action is at the double-stranded helix DNA in cell nuclei. ${ }^{11}$ Therefore, to endow a drug carrier with active targeting ability, a bioactive ligand must be available on the carrier surface for efficient chemical ligation of the ligand and its receptor. The cellular uptake of the three doxorubicin formulations in HepG2 cells was evaluated by confocal laser scanning microscopy, and the representative images of HepG2 cells are shown in Figure 7. All three doxorubicin formulations had an equivalent doxorubicin concentration of $5 \mu \mathrm{g} / \mathrm{mL}$. It can be seen from Figure 7 that fluorescent doxorubicin was visualized in HepG2 cells treated with the three doxorubicin formulations. However, the detected fluorescence in cells treated with doxorubicin-loaded PEG-b-PLMA micelles was much weaker than that in the cells treated with doxorubicin-loaded targeted micelles, indicating that doxorubicin-loaded GalPEG-b-PLMA micelles were more efficiently internalized by HepG2 cells. The result confirmed that galactose-modified micelles could be selectively uptaken by HepG2 cells via asialoglycoprotein receptor-mediated endocytosis.

\section{Conclusion}

New amphiphilic PEG-PLMA block copolymer micelles were successfully developed for targeted delivery of poorly soluble doxorubicin to HepG 2 cells. The polymer micelles, composed of a PEG block as the hydrophilic shell and a PLMA block with pendant carboxylic groups as the hydrophobic core, could effectively incorporate doxorubicin through the combination of hydrophobic and electrostatic interactions between doxorubicin and PLMA. The critical micelle concentration value, drug loading content, and encapsulation efficiency of doxorubicin in the PEG-PLMA micelles were about $1 \mu \mathrm{g} / \mathrm{mL}, 8 \mathrm{wt} \%$, and $83 \%$, respectively. The doxorubicin-loaded or -nonloaded micelles exhibited a smooth surface and spherical shape, and had an average size of less than $100 \mathrm{~nm}$. The micelles showed $\mathrm{pH}$-responsive release behavior, ie, doxorubicin release at mildly acidic $\mathrm{pH} 4.5$ and $\mathrm{pH} 5.5$ was much faster than that at physiologic $\mathrm{pH}$ 7.4. The $\mathrm{pH}$-responsive release behavior is closely related to the presence of carboxyl groups in the PLMA block. Gal-PEG-b-PLMA micelles could be selectively taken up by HepG2 cells through asialoglycoprotein receptor-mediated endocytosis, and exhibited much higher 
cytotoxicity than ungalactosylated micelles. The doxorubicin-loaded micelles could produce $\mathrm{S}$ cell cycle arrest in HepG2 cells. Therefore, the pH-responsive PEG-b-PLMA micelles are promising nanovehicles for targeted delivery of poorly soluble anticancer drugs.

\section{Acknowledgment}

This research was supported by grants from the National Natural Science Foundation of China (No. 50773062 and No. 30830099), the Natural Science Basic Research Plan in Shaanxi Province of China (No. SJ08-ZT12), and the Fundamental Research Funds for the Central Universities (No. XJJ20100115).

\section{Disclosure}

The authors report no conflicts of interest in this work.

\section{References}

1. Yang XL, Zou P, Yao J, et al. Proteomic dissection of cell type-specific $\mathrm{H} 2 \mathrm{AX}$-interacting protein complex associated with hepatocellular carcinoma. J Proteome Res. 2010;9:1402-1415.

2. Huynh H. Molecularly targeted therapy in hepatocellular carcinoma. Biochem Pharmacol. 2010;80:550-560.

3. Xu ZH, Chen LL, Gu WW, et al. The performance of docetaxelloaded solid lipid nanoparticles targeted to hepatocellular carcinoma. Biomaterials. 2009;30:226-232.

4. Fiume L, Baglioni M, Bolondi L, Farina C, Stefano GD. Doxorubicin coupled to lactosaminated human albumin: A hepatocellular carcinoma targeted drug. Drug Discov Today. 2008;13:1002-1009.

5. Zhang J, Chen XG, Sun GZ, Huang L, Cheng XJ. Effect of molecular weight on the oleoyl-chitosan nanoparticles as carriers for doxorubicin. Colloids Surf B Biointerfaces. 2010;77:125-130.

6. Musacchio T, Toniutti M, Kautz R, Torchilin VP. ${ }^{1} \mathrm{H}$ NMR detection of mobile lipids as a marker for apoptosis: The case of anticancer drug-loaded liposomes and polymeric micelles. Mol Pharm. 2009;6: 1876-1882.

7. Wang JR, Xu TW. Facile construction of multivalent targeted drug delivery system from Boltorn ${ }^{\circledR}$ series hyperbranched aliphatic polyester and folic acid. Polym Adv Technol. 2009; doi: 10.1002/pat.1535.

8. Kurtoglu YE, Mishra MK, Kannan S, Kannan RM. Drug release characteristics of PAMAM dendrimer-drug conjugates with different linkers. Int J Pharm. 2010;384:189-194.

9. Zerrin S, Nilufer Y, Tamer B. Investigation of pluronic and PEG-PE micelles as carriers of meso-tetraphenyl porphine for oral administration. Int J Pharm. 2007;332:161-167.

10. Chen W, Meng FH, Li F, Ji SJ, Zhong ZY. pH-responsive biodegradable micelles based on acid-labile polycarbonate hydrophobe: Synthesis and triggered drug release. Biomacromolecules. 2009;10:1727-1735.

11. Li XM, Ding LY, Xu YL, Wang YL, Ping QN. Targeted delivery of doxorubicin using stealth liposomes modified with transferring. Int J Pharm. 2009;373:116-123.

International Journal of Nanomedicine

\section{Publish your work in this journal}

The International Journal of Nanomedicine is an international, peerreviewed journal focusing on the application of nanotechnology in diagnostics, therapeutics, and drug delivery systems throughout the biomedical field. This journal is indexed on PubMed Central, MedLine, CAS, SciSearch ${ }^{\circledR}$, Current Contents ${ }^{\circledR} /$ Clinical Medicine,
12. Zhang C, Gao SJ, Jiang W, et al. Targeted minicircle DNA delivery using folate-poly(ethyleneglycol)-polyethylenimine as non-viral carrier. Biomaterials. 2010;31:6075-6086.

13. Mei H, Shi W, Pang ZQ, et al. EGFP-EGF1 protein-conjugated PEGPLA nanoparticles for tissue factor targeted drug delivery. Biomaterials. 2010;31:5619-5626.

14. Prabaharan M, Grailer JJ, Pilla S, Steeber DA, Gong SQ. Amphiphilic multi-arm-block copolymer conjugated with doxorubicin via $\mathrm{pH}$-sensitive hydrazone bond for tumor-targeted drug delivery. Biomaterials. 2009;30:5757-5766.

15. Patil Y, Sadhukha T, Ma L, Panyam J. Nanoparticle-mediated simultaneous and targeted delivery of paclitaxel and tariquidar overcomes tumor drug resistance. J Control Release. 2009;136:21-29.

16. Kikkeri R, Lepenies B, Adibekian A, Laurino P, Seeberger PH. In vitro imaging and in vivo liver targeting with carbohydrate capped quantum dots. J Am Chem Soc. 2009;131:2110-2112.

17. Higuchi Y, Oka M, Kawakami S, Hashida M. Mannosylated semiconductor quantum dots for the labeling of macrophages. J Control Release. 2008;125:131-136.

18. Wang Z, Chui WK, Ho PC. Design of a multifunctional PLGA nanoparticulate drug delivery system: Evaluation of its physicochemical properties and anticancer activity to malignant cancer cells. Pharm Res. 2009;26:1162-1171.

19. Liu Y, Wang W, Wang J, et al. Blood compatibility evaluation of poly(d,1lactide-co-beta-malic acid) modified with the GRGDS sequence. Colloids Surf B Biointerfaces. 2010;75:370-376.

20. He B, Poon YF, Feng J, Chan-Park MB. Synthesis and characterization of functionalized biodegradable poly(DL-lactide-co-RS- $\beta$-malic acid). J Biomed Mater Res. 2008;87A:254-263.

21. Wang L, Neoh KG, Kang ET, Shuter B, Wang SC. Biodegradable magnetic-fluorescent magnetite/poly(dl-lactic acid-co- $\alpha, \beta$-malic acid) composite nanoparticles for stem cell labeling. Biomaterials. 2010;31: 3502-3511.

22. Coulembier O, Degée P, Barbaud C, Guérin P, Dubois P. New amphiphilic graft copolymer based on poly( $\beta$-malic acid): Synthesis and characterization. Polym Bull. 2004;51:365-372.

23. He B, Wan YQ, Bei JZ, Wang SG. Synthesis and cell affinity of functionalized poly(1-lactide-co- $\beta$-malic acid) with high molecular weight. Biomaterials. 2004;25:5239-5247.

24. Zerrin S, Nilufer Y, Tamer B. Preparation and characterization of polymeric micelles for solubilization of poorly soluble anticancer drugs. Eur J Pharm Biopharm. 2006;64:261-268.

25. Wang Y, Xin DC, Liu KJ, Zhu MQ, Xiang JN. Heparin-paclitaxel conjugates as drug delivery system: Synthesis, self-assembly property, drug release, and antitumor activity. Bioconjug Chem. 2009;20:2214-2221.

26. Hatakeyama H, Akita H, Maruyama K, Suhara T, Harashima H. Factors governing the in vivo tissue uptake of transferrin-coupled polyethylene glycol liposomes in vivo. Int J Pharm. 2004;281:25-33.

27. Kim HS, Lee YS, Kim DK. Doxorubicin exerts cytotoxic effects through cell cycle arrest and fas-mediated cell death. Pharmacology. 2009;84:300-309.

28. Wang Q, Zhang L, Hu W, et al. Norcantharidin-associated galactosylated chitosan nanoparticles for hepatocyte-targeted delivery. Nanomedicine. 2010;6:371-381.

29. Cao Y, Gu Y, Ma H, et al. Self-assembled nanoparticle drug delivery systems from galactosylated polysaccharide-doxorubicin conjugate loaded doxorubicin. Int J Biol Macromol. 2010;46:245-249.

\section{Dovepress}

Journal Citation Reports/Science Edition, EMBase, Scopus and the Elsevier Bibliographic databases. The manuscript management system is completely online and includes a very quick and fair peer-review system, which is all easy to use. Visit http://www.dovepress.com/ testimonials.php to read real quotes from published authors. 